\title{
Set-valued Orthogonal Additivity
}

\author{
Justyna Sikorska
}

Received: 23 November 2014 / Accepted: 9 February 2015 / Published online: 26 February 2015

(C) The Author(s) 2015. This article is published with open access at Springerlink.com

\begin{abstract}
We study the set-valued Cauchy equation postulated for orthogonal vectors. We give its general solution as well as we look for selections of functions satisfying the equation.
\end{abstract}

Keywords Orthogonally additive equation · Quadratic equation · Selection · Set-valued function · Hukuhara difference · Hausdorff distance

Mathematics Subject Classification (2010) 39B55 - 39B52 $\cdot 54$ C60 $\cdot 54$ C65 26 E25

\section{Introduction}

We call function $f: X \rightarrow Y$ orthogonally additive if it satisfies a conditional functional equation

$$
f(x+y)=f(x)+f(y) \text { for all } x, y \in X \text { with } x \perp y .
$$

In the standard settings $X$ is considered to be a real inner product space with the orthogonality relation given by means of the inner product and $Y$ is an Abelian group. However, we may introduce an abstract orthogonality relation in any at least two-dimensional linear space, defining a so called orthogonality space.

The orthogonal additivity (1) has wide applications both inside and outside mathematics. With help of it we can give, e.g., several characterizations of inner product spaces among normed spaces as well as of Hilbert spaces among Banach spaces (see Rätz [25] or Sikorska [29] for more reference items).

Equation (1) has its applications in physics, in the theory of ideal gas (see Aczél \& Dhombres [1], Truesdell \& Muncaster [32], Arkeryd \& Cercignani [2]). In the threedimensional Euclidean space, by means of (1) we obtain the formula for the distribution law of velocities in an ideal gas at a fixed temperature.

J. Sikorska $(\bowtie)$

Institute of Mathematics, University of Silesia, Bankowa 14, 40-007 Katowice, Poland

e-mail: justyna.sikorska@us.edu.pl 
There are also applications of (1) in actuarial mathematics in a premium calculation principle (see Heijnen \& Goovaerts [11]): it was shown, namely, that the variance principle is the only covariance-additive premium principle.

We start with the following definition of the orthogonality space (see Gudder \& Strawther [10], Rätz [22]).

Let $X$ be a real linear space with $\operatorname{dim} X \geq 2$ and let $\perp$ be a binary relation on $X$ such that

(01) $x \perp 0$ and $0 \perp x$ for all $x \in X$;

(02) if $x, y \in X \backslash\{0\}$ and $x \perp y$, then $x$ and $y$ are linearly independent;

(03) if $x, y \in X$ and $x \perp y$, then for all $\alpha, \beta \in \mathbb{R}$ we have $\alpha x \perp \beta y$;

(04) for any two-dimensional subspace $P$ of $X$, for every $x \in P$ and for every $\lambda>0$, there exists $y \in P$ such that $x \perp y$ and $x+y \perp \lambda x-y$.

An ordered pair $(X, \perp)$ is called an orthogonality space.

The notion of the orthogonality space covers the case of an inner product space with the classical orthogonality as well as an arbitrary real normed linear space with the so called Birkhoff orthogonality. But it is also the case with the "trivial" orthogonality defined on a linear space by (01) and the condition that two nonzero vectors are orthogonal if and only if they are linearly independent.

Solutions of (1) are known (see Rätz [22], and also Rätz [23, 24], Rätz \& Szabó [26], Baron \& Rätz [4] and Baron \& Volkmann [5]). Before giving the form of solutions, let us recall that a mapping $q$ defined on a group is called quadratic if for all $x$ and $y$ from the domain, $q(x+y)+q(x-y)=2 q(x)+2 q(y)$.

Theorem 1.1 Let $(X, \perp)$ be an orthogonality space and $(Y,+)$ be an Abelian group. Every orthogonally additive function $f: X \rightarrow Y$ has the form $f=a+q$, where $a$ is additive and $q$ is quadratic (and orthogonally additive).

Theorem 1.2 Let $(X,\|\cdot\|)$ be a real inner product space with $\operatorname{dim} X \geq 2$ and let $(Y,+)$ be an Abelian group. Then $f: X \rightarrow Y$ is orthogonally additive if and only if there exist additive mappings $a: X \rightarrow Y$ and $b: \mathbb{R} \rightarrow Y$ such that $f(x)=a(x)+b\left(\|x\|^{2}\right)$ for all $x \in X$.

Studies of set-valued additive functions have already a pretty long history (see, e.g., Rådström [21], Henney [12, 13], Godini [9], Nikodem [16, 17]). Looking for the selections of such functions is interesting due to their applications, among others, in stability considerations (see, e.g., [3]).

The main aim of this paper is to combine the above strands of research and to study the conditional equation

$$
F(x+y)=F(x)+F(y) \text { for all } x, y \in X \text { with } x \perp y,
$$

where $F$ maps an orthogonality space $X$ into the family of non-empty subsets of a Fréchet space or, more generally, a topological vector space $Y$.

In the paper we will use the following notations. For a topological vector space $Y$, let $c(Y)$ denote the collection of all nonempty compact subsets of $Y, c c(Y)$ - the family of all convex members of $c(Y)$, and $\operatorname{bcl}(Y)$ - the collection of all nonempty, closed and bounded subsets of $Y$.

While speaking generally about a topological vector space $Y$ we will assume that it satisfies $\mathcal{T}_{0}$ separation axiom. This implies already that the space considered is not only Hausdorff but also regular. 


\section{Solutions of the Equation}

We start our considerations with solving (2) in the class of functions defined on an orthogonality space and with values in the family of nonempty compact and convex elements of a metric linear space. The main steps used for solving the equation of orthogonal additivity in a single-valued case are: splitting the function into its odd and even parts - both also orthogonally additive, looking for the solutions of each part separately, and representing the final result as the sum of the so obtained partial solutions.

While solving the equation of orthogonal additivity for set-valued functions we cannot use the same methods directly.

Indeed, if for all $x \in X$,

$$
G(x):=\frac{1}{2}(F(x)+F(-x)) \text { and } H(x):=\frac{1}{2}(F(x)-F(-x))
$$

(with "normal" algebraic difference), then, in general,

$$
F(x) \neq G(x)+H(x) .
$$

For that reason we will use another difference, namely the so called Hukuhara difference (see Hukuhara [15]), which however not always exists.

Let $Y$ be a real topological vector space and let $A, B, C$ be elements of $\operatorname{cc}(Y)$. We say that a set $C$ is the Hukuhara difference of $A$ and $B$, i.e., $C=A-B$, whenever $B+C=A$. If this difference exists, then it is unique (by Corollary 2.1 below).

In what follows we give some notations and present results which will be used in the sequel.

Lemma 2.1 (Rådström's cancelation law) Assume that $A, B, C$ are subsets of a topological vector space $Y$ such that $B$ is closed and convex, $C$ is bounded, nonempty, and $A+C \subset$ $B+C$. Then $A \subset B$.

The above law has been formulated by Rådström's [20] in the case where $Y$ is a normed space but the proof given there is valid in topological vector spaces (cf., Smajdor [30], Urbański [33]).

Corollary 2.1 Assume that $A, B, C$ are subsets of a topological vector space $Y$ such that $A$ and $B$ are closed and convex, $C$ is bounded, nonempty, and $A+C=B+C$. Then $A=B$.

If $(Y, d)$ is a metric space, on the set of all nonempty closed and bounded subsets of $Y$ we define the Hausdorff distance as follows. For any $A, B \in \operatorname{bcl}(Y)$,

$$
h(A, B):=\max \left\{\sup _{x \in A} d(x, B), \sup _{y \in B} d(A, y)\right\},
$$

where $d(x, B):=\inf \{d(x, y): y \in B\}$. Or, equivalently,

$$
h(A, B)=\inf \left\{\varepsilon>0: A \subset B_{\varepsilon} \text { and } B \subset A_{\varepsilon}\right\},
$$

where $A_{\varepsilon}=\cup_{a \in A} K(a, \varepsilon)$ and $K(a, \varepsilon)=\{x \in Y: d(a, x) \leq \varepsilon\}$. In the case of (translation) invariant metric on a linear space, $A_{\varepsilon}=A+K(\varepsilon)$, where $K(\varepsilon)$ is simply a closed ball of radius $\varepsilon$ centered at the origin. It can be shown that the Hausdorff distance between two elements of $\operatorname{bcl}(Y)$ is the uniform distance between their associated distance functionals [6, Section 3.2]. The space $b c l(Y)$ equipped with the Hausdorff distance forms a metric space. 
It is known that if $(Y, d)$ is complete, so do $b c l(Y), c(Y)$ and $c c(Y)$, if considered with the Hausdorff metric (see, e.g., Castaing \& Valadier [7] or Beer [6]).

In the sequel, we list some properties of the Hausdorff distance.

Lemma 2.2 Assume that $(Y, d)$ is a metric linear space. Then

(a) for any $A, B, C \in \operatorname{bcl}(Y), h(A+C, B+C) \leq h(A, B)$;

(b) for all $A_{n}, B_{n}, A, B \in \operatorname{bcl}(Y), n \in \mathbb{N}$, if $A_{n} \rightarrow A$ and $B_{n} \rightarrow B$, then $A_{n}+B_{n} \rightarrow$ $A+B$

(c) if $d$ is invariant, then for any $A, B \in \operatorname{bcl}(Y)$ and some $\varepsilon>0$,

$h(A, B)<\varepsilon \quad \Longrightarrow \quad(A \subset B+K(\varepsilon)$ and $B \subset A+K(\varepsilon)) \quad \Longrightarrow \quad h(A, B)<2 \varepsilon ;$

(d) if $A \in \operatorname{bcl}(Y),\left(\alpha_{n}\right)_{n \in \mathbb{N}}$ is a sequence of real numbers converging to $\alpha \in \mathbb{R}$ and $d$ is invariant, then $\alpha_{n} A \rightarrow \alpha A$.

Proof For (d), take $A \in \operatorname{bcl}(Y)$, a sequence $\alpha_{n} \rightarrow \alpha \in \mathbb{R}, n \in \mathbb{N}$, and fix $\varepsilon>0$. Let $U$ be a balanced and symmetric neighbourhood of zero such that $U \subset K(\varepsilon)$. By the boundedness of $A$, there exists $\mu>0$ such that $A \subset \mu U$. By the convergence of $\left(x_{n}\right)_{n \in \mathbb{N}}$, there exists $n_{0} \in \mathbb{N}$ such that $\xi_{n}:=\alpha_{n}-\alpha$ satisfy $\left|\xi_{n}\right|<\frac{1}{\mu}$ for all $n \geq n_{0}$.

For any $n \geq n_{0}$ we have

$a_{n} A=\left(\alpha+\xi_{n}\right) A \subset \alpha A+\xi_{n} A \subset \alpha A+\xi_{n} \mu U=\alpha A+\left|\xi_{n}\right| \mu U \subset \alpha A+U \subset \alpha A+K(\varepsilon)$.

Analogously, we obtain

$$
\alpha A \subset \alpha_{n} A+K(\varepsilon),
$$

which, by (c), finishes the proof of (d).

By Lemma 2.2(c), we see in fact that the convergence of a sequence of sets with respect to the Hausdorff metric can be equivalently defined as: $A_{n} \rightarrow A$ if and only if for every neighbourhood $V$ of zero in $Y$ (or element of a basis of neighbourhoods of zero) there exists $n_{0} \in \mathbb{N}$ such that $A_{n} \subset A+V$ and $A \subset A_{n}+V$ for every $n \geq n_{0}$. By this way, we say also that $\left(A_{n}\right)_{n \in \mathbb{N}}$ is a Cauchy sequence if for every neighbourhood $V$ of zero in $Y$ there exists $n_{0} \in \mathbb{N}$ such that $A_{n} \subset A_{m}+V$ and $A_{m} \subset A_{n}+V$ for all $m, n \geq n_{0}$.

The proof of the next lemma in the case when $Y$ is a normed linear space, was given by Piszczek in [19].

Lemma 2.3 Assume that $(Y, d)$ is a Fréchet space (locally convex, complete metric linear space with an invariant metric; see, e.g., Rolewicz [27]). If $A_{n}, B_{n}, A, B \in c c(Y), n \in \mathbb{N}$, $A_{n} \rightarrow A, B_{n} \rightarrow B$ and there exist the Hukuhara differences $A_{n}-B_{n}$ for all $n \in \mathbb{N}$, then there exists the Hukuhara difference $A-B$ and $A_{n}-B_{n} \rightarrow A-B$.

Proof Let $C_{n}:=A_{n}-B_{n}$ for all $n \in \mathbb{N}$, whence $A_{n}=B_{n}+C_{n}, n \in \mathbb{N}$. Let $U$ be a neighbourhood of zero in $Y$. Since $Y$ is locally convex, there exist convex neighbourhoods of zero $W, W_{1}$ such that $\bar{W} \subset W_{1} \subset U$. Further, there exists a neighbourhood $V$ of zero such that $V+V \subset W$. Since $A_{n} \rightarrow A$ and $B_{n} \rightarrow B$ then there exists $n_{0} \in \mathbb{N}$ such that for all $n, m \geq n_{0}$,

$$
A_{n} \subset A_{m}+V, \quad A_{m} \subset A_{n}+V, \quad B_{n} \subset B_{m}+V, \quad B_{m} \subset B_{n}+V .
$$

Take $n, m \geq n_{0}$. We have the following inclusions

$$
A_{n}+B_{m} \subset A_{m}+B_{m}+V, A_{m}+B_{m} \subset A_{m}+B_{n}+V,
$$




$$
B_{n}+C_{n}+B_{m} \subset B_{m}+C_{m}+B_{m}+V, \quad B_{m}+C_{m}+B_{m} \subset B_{m}+C_{m}+B_{n}+V,
$$

whence,

$$
B_{n}+C_{n}+B_{m} \subset B_{m}+C_{m}+B_{n}+V+V \subset B_{m}+C_{m}+B_{n}+\bar{W} .
$$

By Lemma 2.1,

$$
C_{n} \subset C_{m}+\bar{W} \subset C_{m}+U .
$$

Analogously, we obtain $C_{m} \subset C_{n}+U$. This means that $\left(C_{n}\right)$ is a Cauchy sequence in $c c(Y)$, and so, $\left(C_{n}\right)$ is convergent, say to $C \in \operatorname{cc}(Y)$.

On the other side, for a fixed neighbourhood $U$ of zero and $V$ such that $V+V \subset U$ there exists $n_{0} \in \mathbb{N}$ such that for all $n \geq n_{0}$,

$$
C_{n} \subset C+V, \quad C \subset C_{n}+V, \quad B_{n} \subset B+V, \quad B \subset B_{n}+V .
$$

Hence,

$$
B_{n}+C_{n} \subset B+C+U, \quad B+C \subset B_{n}+C_{n}+U .
$$

It means that $B_{n}+C_{n} \rightarrow B+C$. But $B_{n}+C_{n}=A_{n} \rightarrow A$. Consequently, since $c c(Y)$ is a Hausdorff space, $B+C=A$, and $C=A-B$.

In what follows we present the main result of the paper concerning the general solutions of (2).

Theorem 2.1 Let $(X, \perp)$ be an orthogonality space and $(Y, d)$ be a Fréchet space. If $F: X \rightarrow c c(Y)$ satisfies (2), then there exist an additive function $a: X \rightarrow Y$ and $a$ quadratic function $Q: X \rightarrow c c(Y)$, i.e., satisfying for all $x, y \in X$ condition $Q(x+y)+$ $Q(x-y)=2 Q(x)+2 Q(y)$, which is orthogonally additive, such that $F=a+Q$. Moreover, such representation is unique.

Proof Put $x=y=0$ in (2). Since $F(0)$ is bounded, on account of Corollary 2.1 immediately we get

$$
F(0)=\{0\} .
$$

Take $x \in X$. There exists $y \in X$ such that $x \perp y$ and $x+y \perp x-y$. By (2) and the properties of the orthogonality relation we obtain (cf., [8] or [28] for more general results)

$$
\begin{aligned}
F(2 x) & =F(x+y)+F(x-y) \\
F(x+y) & =F(x)+F(y) \\
F(x-y) & =F(x)+F(-y) \\
F(-x-y)+F(-x+y) & =F(-2 x) \\
F(-x)+F(-y) & =F(-x-y) \\
F(y) & =F\left(\frac{x+y}{2}\right)+F\left(\frac{-x+y}{2}\right) \\
F(-y) & =F\left(\frac{-x-y}{2}\right)+F\left(\frac{x-y}{2}\right) \\
F\left(\frac{-x-y}{2}\right)+F\left(\frac{-x+y}{2}\right) & =F(-x) \\
F\left(\frac{x+y}{2}\right)+F\left(\frac{x-y}{2}\right) & =F(x) .
\end{aligned}
$$


Multiplying first three from the above equations by 3 and last four equations by 2, and summing up all ten equalities side by side, by convexity of the sets we derive

$$
\begin{aligned}
3 F(2 x) & +3 F(x+y)+3 F(x-y)+F(-x+y)+F(-x-y) \\
& +F(-x)+F(y)+F(-x)+F(-y)+2 F(y)+2 F(-y) \\
& +2 F\left(\frac{-x+y}{2}\right)+2 F\left(\frac{-x-y}{2}\right)+2 F\left(\frac{x+y}{2}\right)+2 F\left(\frac{x-y}{2}\right) \\
& =3 F(x+y)+3 F(x-y)+3 F(x)+2 F(y)+F(y)+3 F(x)+2 F(-y)+F(-y) \\
& +F(-2 x)+F(-x+y)+F(-x-y)+2 F\left(\frac{-x+y}{2}\right)+2 F\left(\frac{x+y}{2}\right) \\
& +2 F\left(\frac{-x-y}{2}\right)+2 F\left(\frac{x-y}{2}\right)+2 F(-x)+2 F(x) .
\end{aligned}
$$

On account of Corollary 2.1, we have

$$
3 F(2 x)=F(-2 x)+8 F(x), \quad x \in X .
$$

In the next step, using some ideas from Smajdor [31], we will prove that there exists the Hukuhara difference $F(x)-F(-x)$. First, by induction we will prove that for every $n \in \mathbb{N}$ we have

$$
\frac{2^{n}+1}{2 \cdot 4^{n}} F(x)=F\left(\frac{x}{2^{n}}\right)+\frac{2^{n}-1}{2 \cdot 4^{n}} F(-x), \quad x \in X .
$$

Validity of (6) for $n=1$, i.e.,

$$
\frac{3}{8} F(x)=F\left(\frac{x}{2}\right)+\frac{1}{8} F(-x), \quad x \in X,
$$

follows from (5) (and the convexity of sets). By validity of (6) for a fixed $n$, and by (7) we obtain

$$
\begin{aligned}
\frac{2^{n}+1}{2 \cdot 4^{n}} F\left(\frac{x}{2}\right) & =F\left(\frac{x}{2^{n+1}}\right)+\frac{2^{n}-1}{2 \cdot 4^{n}} F\left(-\frac{x}{2}\right) \\
\frac{2^{n}+1}{2 \cdot 4^{n}} \cdot \frac{3}{8} F(x) & =\frac{2^{n}+1}{2 \cdot 4^{n}} F\left(\frac{x}{2}\right)+\frac{2^{n}+1}{2 \cdot 4^{n}} \cdot \frac{1}{8} F(-x) \\
\frac{2^{n}-1}{2 \cdot 4^{n}} F\left(-\frac{x}{2}\right)+\frac{2^{n}-1}{2 \cdot 4^{n}} \cdot \frac{1}{8} F(x) & =\frac{2^{n}-1}{2 \cdot 4^{n}} \cdot \frac{3}{8} F(-x) .
\end{aligned}
$$

Summing up the above equalities, by Corollary 2.1 we get

$$
\begin{aligned}
& \frac{2^{n}+1}{2 \cdot 4^{n}} F\left(\frac{x}{2}\right)+\frac{2^{n}+1}{2 \cdot 4^{n}} \cdot \frac{3}{8} F(x)+\frac{2^{n}-1}{2 \cdot 4^{n}} F\left(-\frac{x}{2}\right)+\frac{2^{n}-1}{2 \cdot 4^{n}} \cdot \frac{1}{8} F(x) \\
= & F\left(\frac{x}{2^{n+1}}\right)+\frac{2^{n}-1}{2 \cdot 4^{n}} F\left(-\frac{x}{2}\right)+\frac{2^{n}+1}{2 \cdot 4^{n}} F\left(\frac{x}{2}\right)+\frac{2^{n}+1}{2 \cdot 4^{n}} \cdot \frac{1}{8} F(-x)+\frac{2^{n}-1}{2 \cdot 4^{n}} \cdot \frac{3}{8} F(-x),
\end{aligned}
$$

whence

$$
\frac{2^{n+1}+1}{2 \cdot 4^{n+1}} \cdot F(x)=F\left(\frac{x}{2^{n+1}}\right)+\frac{2^{n+1}-1}{2 \cdot 4^{n+1}} F(-x), \quad x \in X,
$$

so (6) holds for all $n \in \mathbb{N}$. Consequently, for all $n \in \mathbb{N}$ and all $x \in X$ there exists the Hukuhara difference

$$
F\left(\frac{x}{2^{n}}\right)=\frac{2^{n}+1}{2 \cdot 4^{n}} F(x)-\frac{2^{n}-1}{2 \cdot 4^{n}} F(-x),
$$

and we have

$$
2^{n} F\left(\frac{x}{2^{n}}\right)=\frac{2^{n}+1}{2 \cdot 2^{n}} F(x)-\frac{2^{n}-1}{2 \cdot 2^{n}} F(-x), \quad x \in X, n \in \mathbb{N} .
$$


By Lemma 2.3 and Lemma 2.2, there exists the Hukuhara difference $\frac{1}{2} F(x)-\frac{1}{2} F(-x)$ for all $x \in X$. Define $H(x):=\frac{1}{2}(F(x)-F(-x))$ for all $x \in X$. We have $F(x)=$ $2 H(x)+F(-x)$ and $F(-x)=2 H(-x)+F(x)$, whence

$$
F(x)=2 H(x)+2 H(-x)+F(x), \quad x \in X .
$$

On account of Corollary 2.1,

$$
2 H(x)+2 H(-x)=\{0\}, \quad x \in X,
$$

and $H$ is a single valued function

$$
H(x)=\{a(x)\}, \quad x \in X .
$$

We will show that $a$ is additive. For this end, fix $x, y \in X, x \perp y$. We have

$$
\begin{aligned}
\{a(x)\}+\{a(y)\} & =H(x)+H(y)=\frac{1}{2}(F(x)-F(-x))+\frac{1}{2}(F(y)-F(-y)) \\
& =\frac{1}{2}(F(x)+F(y))-\frac{1}{2}(F(-x)+F(-y))=\frac{1}{2} F(x+y)-\frac{1}{2} F(-x-y) \\
& =\frac{1}{2}(F(x+y)-F(-x-y))=H(x+y)=\{a(x+y)\} .
\end{aligned}
$$

Hence, $a$ is orthogonally additive and odd, so it is additive (see, e.g., Rätz [22]).

Define $Q(x):=\frac{1}{2}(F(x)+F(-x))$ for all $x \in X$. $Q$ is an even, orthogonally additive function. We will show that it is quadratic, and using a procedure analogous to one used by Rätz [22] (see also Rätz [23, 24], Rätz \& Szabó [26]), we will proceed with four steps.

(a) If $x+y \perp x-y$, then $Q(x)=Q(y)$.

Fix $x, y \in X, x+y \perp x-y$. Then

$$
Q(x)=Q\left(\frac{x+y}{2}\right)+Q\left(\frac{x-y}{2}\right)=Q\left(\frac{x+y}{2}\right)+Q\left(\frac{y-x}{2}\right)=Q(y) .
$$

(b) $Q(2 x)=4 Q(x), x \in X$.

Fix $x \in X$; there exists $y \in X$ such that $x \perp y$ and $x+y \perp x-y$. Then, by (a),

$$
Q(2 x)=Q(x+y)+Q(x-y)=Q(x))+Q(y)+Q(x)+Q(-y)=4 Q(x) .
$$

(c) $Q(\alpha x+\beta x)+Q(\alpha x-\beta x)=2 Q(\alpha x)+2 Q(\beta x), x \in X, \alpha, \beta \in \mathbb{R}$.

Fix $x \in X$ and $\alpha, \beta \in \mathbb{R}$. There exists $y \in X$ such that $x \perp y$ and $x+y \perp x-y$. We have

$$
\begin{aligned}
Q((\alpha+\beta) x) & +Q((\alpha-\beta) y)=Q((\alpha+\beta) x+(\alpha-\beta) y)=Q(\alpha(x+y)+\beta(x-y)) \\
& =Q(\alpha(x+y))+Q(\beta(x-y))=Q(\alpha x)+Q(\alpha y)+Q(\beta x)+Q(\beta y)
\end{aligned}
$$

Set in the above $\beta:=\alpha$. Then, since by (4), $Q(0)=\{0\}$, we have

$$
Q(2 \alpha x)=2 Q(\alpha x)+2 Q(\alpha y),
$$

and, by (b) we obtain $Q(\alpha x)=Q(\alpha y)$. Consequently, the substitution of the last equality into (9) gives (c).

(d) $Q(x+y)+Q(x-y)=2 Q(x)+2 Q(y), x, y \in X$.

Take $x, y \in X$. If $x$ and $y$ are linearly dependent, the equality holds by (c). Assume $x$ and $y$ are linearly independent. There exist $u, v \in X$ such that $u \perp v, u+v \perp u-v$ 
and $\operatorname{lin}\{u, v\}=\operatorname{lin}\{x, y\}$. Then $x=\alpha u+\beta v, y=\gamma u+\delta v$ for some $\alpha, \beta, \gamma, \delta \in \mathbb{R}$. We have

$$
\begin{aligned}
Q(x+y)+Q(x-y) & =Q(\alpha u+\beta v+\gamma u+\delta v)+Q(\alpha u+\beta v-\gamma u-\delta v) \\
& =Q((\alpha+\gamma) u)+Q((\beta+\delta) v)+Q((\alpha-\gamma) u)+Q((\beta-\delta) v) \\
& \stackrel{(c)}{=} 2 Q(\alpha u)+2 Q(\gamma u)+2 Q(\beta v)+2 Q(\delta v) \\
& =2 Q(\alpha u+\beta v)+2 Q(\gamma u+\delta v)=2 Q(x)+2 Q(y) .
\end{aligned}
$$

Consequently,

$$
a(x)+Q(x)=\{a(x)\}+Q(x)=\frac{1}{2}(F(x)-F(-x))+\frac{1}{2}(F(x)+F(-x))=F(x),
$$

for all $x \in X$.

For proving the uniqueness of the representation, let an additive function $b: X \rightarrow Y$, and a quadratic function $R: X \rightarrow c c(Y)$ be second pair of functions such that $F=b+R$. Then

$$
\begin{gathered}
F(x)=a(x)+Q(x)=b(x)+R(x) \\
a\left(2^{n} x\right)+Q\left(2^{n} x\right)=b\left(2^{n} x\right)+R\left(2^{n} x\right) \\
\frac{1}{2^{n}} a(x)+Q(x)=\frac{1}{2^{n}} b(x)+R(x), \quad n \in \mathbb{N}, x \in X,
\end{gathered}
$$

whence, $Q=R$ and, on account of Corollary 2.1 and (10), $a=b$. This finishes the proof.

Theorem 2.2 Let $(X,\|\cdot\|)$ be an inner product space and $(Y, d)$ be a Fréchet space. Function $F: X \rightarrow c c(Y)$ satisfies (2) if and only if there exist additive functions $a: X \rightarrow Y$ and $B:[0, \infty) \rightarrow c c(Y)$ such that

$$
F(x)=a(x)+B\left(\|x\|^{2}\right), \quad x \in X .
$$

Proof It is easy to see that function $F$ of the form (11) is a solution of (2).

By Theorem 2.1 we know that $F=a+Q$, where $a: X \rightarrow Y$ is additive and $Q: X \rightarrow$ $c c(Y)$ is quadratic. In order to finish the proof, it is enough to find the form of the quadratic function $Q$. Take $x, y \in X$ such that $\|x\|=\|y\|$. We have $x+y \perp x-y$ and by (a) in the proof of Theorem 2.1, $Q(x)=Q(y)$, so $Q$ is constant on spheres centered at the origin. Hence, there exists a function $B:[0, \infty) \rightarrow c c(Y)$ such that $Q(x)=B\left(\|x\|^{2}\right)$ for all $x \in X$. Repeating the argument used by Rätz in [22], since $Q$ is orthogonally additive, we derive that $B$ is additive on the positive half-line.

Having solved (2), we may look for selections of such multivalued functions. However, in the next section we will do it in more general settings (cf., Theorem 3.1 and Remark 3.1).

\section{Selections}

In this section we ask whether for a given set-valued orthogonally additive function $F: X \rightarrow c(Y)$, i.e., a function satisfying (2), there exists a single-valued orthogonally additive function $f: X \rightarrow Y$ such that $f(x) \in F(x)$ for every $x \in X$. Such a function $f$ is called a selection for $F$. We apply an approach similar to one used by Smajdor [30] for quadratic functions (see also Smajdor [31]). Another approach of proving the existence of an additive selection of a multi-valued additive function was presented by Nikodem in [17]. In conditional version, however, such approach fails to be used. 
Lemma 3.1 Let $(X, \perp)$ be an orthogonality space and let $Y$ be a topological vector space. If $F: X \rightarrow c(Y)$ satisfies (2) then there exists a minimal (in the sense of inclusion between values for each fixed argument) solution $F_{0}: X \rightarrow c(Y)$ of this equation contained in $F$.

Proof Define

$$
\mathcal{F}:=\{G: X \rightarrow c(Y): G \text { is a solution of (2) and } G \subset F\} .
$$

$\mathcal{F}$ is nonempty since $F \in \mathcal{F}$. Consider the chain $\mathcal{C} \subset \mathcal{F}$. Then $F_{1}(x):=\bigcap_{G \in \mathcal{C}} G(x), x \in$ $X$, is a solution of (2). Indeed, take $x, y \in X$ with $x \perp y$. On account of Lemma 10.4 in [14] (see also Lemma 1.5 in [30]) we have

$$
F_{1}(x)+F_{1}(y)=\bigcap_{G \in \mathcal{C}} G(x)+\bigcap_{G \in \mathcal{C}} G(y)=\bigcap_{G \in \mathcal{C}}(G(x)+G(y))=\bigcap_{G \in \mathcal{C}} G(x+y)=F_{1}(x+y) .
$$

Obviously $F_{1} \subset F$, so $F_{1} \in \mathcal{F}$. By the Kuratowski-Zorn Lemma, there exists a minimal element of $\mathcal{F}$.

Theorem 3.1 Let $(X, \perp)$ be an orthogonality space and let $Y$ be a locally convex linear topological space. If $F: X \rightarrow c(Y)$ satisfies (2), then there exists a selection $f: X \rightarrow Y$ of $F$ satisfying (1).

Proof By Lemma 3.1, there exists a minimal solution $F_{0}$ of (2). We will show that $F_{0}$ is a single-valued function. Suppose, to the contrary, that there exists $x_{0} \in X$ and two different elements $y_{1}, y_{2} \in F_{0}\left(x_{0}\right)$. Space $Y$ is locally convex, so by the Hahn-Banach theorem there exists a continuous linear functional $l \in Y^{*}$ such that $l\left(y_{1}\right) \neq l\left(y_{2}\right)$. Define

$$
G(x):=\left\{y \in F_{0}(x): \max l\left(F_{0}(x)\right)=l(y)\right\}, \quad x \in X .
$$

$G(x)$ is nonempty, compact as a closed subset of a compact set, and $G(x) \subset F_{0}(x)$ for any $x \in X$. Moreover, it is a solution of (2). Indeed, fix $x, y \in X, x \perp y$ and take $u \in$ $G(x)+G(y)$. There exist $v \in G(x)$ and $w \in G(y)$ such that $u=v+w$. Surely, $v \in F_{0}(x)$, $w \in F_{0}(y)$ and $l(v)=\max l\left(F_{0}(x)\right), l(w)=\max l\left(F_{0}(y)\right)$. We have

$$
u=v+w \in F_{0}(x)+F_{0}(y)=F_{0}(x+y),
$$

and

$$
\begin{aligned}
l(u) & =l(v)+l(w)=\max l\left(F_{0}(x)\right)+\max l\left(F_{0}(y)\right) \\
& =\max l\left(F_{0}(x)+F_{0}(y)\right)=\max l\left(F_{0}(x+y)\right),
\end{aligned}
$$

whence, $u \in G(x+y)$. A similar argument can be used to prove the second inclusion. Namely, for a fixed $x, y \in X, x \perp y$ take $u \in G(x+y)$. Then

$$
l(u)=\max l\left(F_{0}(x+y)\right)=\max l\left(F_{0}(x)+F_{0}(y)\right)=\max l\left(F_{0}(x)\right)+\max l\left(F_{0}(y)\right) .
$$

Of course, $u \in F_{0}(x+y)$, and since $F_{0}$ satisfies (2), there exist $v \in F_{0}(x)$ and $w \in F_{0}(y)$ such that $u=v+w$. We have

$$
l(u)=l(v)+l(w) \leq \max l\left(F_{0}(x)\right)+\max l\left(F_{0}(y)\right)=l(u),
$$

whence, $v \in G(x), w \in G(y)$ (if we had, e.g., $v \notin G(x)$, it would follow that $\max l\left(F_{0}(x)\right)>l(v)$, and then from the equality above it would follow that $\max l\left(F_{0}(y)\right)<$ $l(w)$; impossible), and $u \in G(x)+G(y)$. This shows that $G$ is a solution of (2).

Since $G(x) \subset F_{0}(x)$ and $F_{0}$ is a minimal solution of (2) contained in $F$, it is $G=F_{0}$. This gives a contradiction, since, in particular it is not true that both $y_{1}, y_{2} \in G\left(x_{0}\right)$. Indeed, if $y_{1} \in G\left(x_{0}\right)$, then $\max l\left(F_{0}\left(x_{0}\right)\right)=l\left(y_{1}\right) \neq l\left(y_{2}\right)$, whence, $y_{2} \notin G\left(x_{0}\right)$, and the same we 
would get if we supposed $y_{2} \in G\left(x_{0}\right)$. Consequently, the contradiction shows that $F_{0}(=G)$ is single-valued. So, there exists $f: X \rightarrow Y$ satisfying (1) such that $f(x) \in F(x)$ for any $x \in X$

Remark 3.1 In Theorem 3.1 we may assume less, namely $Y$ can be a topological vector space that has sufficiently many linear functionals, i.e., for every $y \in Y$ with $y \neq 0$ there exists a continuous linear functional $l \in Y^{*}$ such that $l(y) \neq 0$. By the Hahn-Banach theorem, every locally convex space has sufficiently many linear functionals.

Remark 3.2 The condition $x \perp y$ in (2) may be changed for more general $(x, y) \in D$ for any $\emptyset \neq D \subset X \times X$ and the respective assertions of Lemma 3.1 and Theorem 3.1 will remain valid.

The selection in Theorem 3.1 is not uniquely determined.

Example 3.1 Let $(X,\|\cdot\|)$ be an inner product space with $\operatorname{dim} X \geq 2$ and let $Y$ be a locally convex space. Let, moreover, $A \in c c(Y)$. Then function $F: X \rightarrow c c(Y)$ defined as $F(x):=\|x\|^{2} A, x \in X$, is set-valued orthogonally additive.

Of course, every function $f: X \rightarrow Y$ given by $f(x):=\|x\|^{2} a, x \in X$, with $a \in A$ is an orthogonally additive selection of $F$.

We finish the section with two easy corollaries.

Corollary 3.1 Let $(X, \perp)$ be an orthogonality space and let $Y$ be a locally convex topological vector space. If $F: X \rightarrow c(Y)$ satisfies (2), then there exist an additive function $a: X \rightarrow Y$ and a quadratic function $q: X \rightarrow Y$, which is orthogonally additive, such that $a(x)+q(x) \in F(x)$ for all $x \in X$.

Corollary 3.2 Let $(X,\|\cdot\|)$ be an inner product space with $\operatorname{dim} X \geq 2$ and let $Y$ be a locally convex topological vector space. If $F: X \rightarrow c(Y)$ satisfies (2), then there exist additive functions $a: X \rightarrow Y$ and $b: \mathbb{R} \rightarrow Y$ such that $a(x)+b\left(\|x\|^{2}\right) \in F(x)$ for all $x \in X$.

In the present section we were checking for the existence of selections of a set-valued orthogonally additive function with values being nonempty compact subsets of a locally convex topological vector space $Y$. It is an open problem whether solutions $F: X \rightarrow \operatorname{cc}(Y)$ of (2) for such a space $Y$ remain of the same form as it was for a Fréchet space.

Conflict of Interest Statement The author declares that she has no conflict of interest.

Open Access This article is distributed under the terms of the Creative Commons Attribution License which permits any use, distribution, and reproduction in any medium, provided the original author(s) and the source are credited.

\section{References}

1. Aczél, J., Dhombres, J.: Functional Equations in Several Variables. Encyclopedia of Mathematics and its Applications, vol. 31. Cambridge University Press (1989) 
2. Arkeryd, L., Cercignani, C.: On a functional equation arising in the kinetic theory of gases. Atti Accad. Naz. Lincei Cl. Sci. Fis. Mat. Natur. Rend. Lincei (9), Mat. Appl. 1, 139-149 (1990)

3. Badora, R., Ger, R., Páles, Zs.: Additive selections and the stability of the Cauchy functional equation. ANZIAM J 44, 323-337 (2003)

4. Baron, K., Rätz, J.: On orthogonally additive mappings on inner product spaces. Bull. Polish Acad. Sci. Math. 43, 187-189 (1995)

5. Baron, K., Volkmann, P.: On orthogonally additive functions. Publ. Math. Debrecen 52, 291-297 (1998)

6. Beer, G.: Topologies on Closed and Closed Convex Sets. Mathematics and its Applications, vol. 268. Kluwer Academic Publishers Group, Dordrecht (1993)

7. Castaing, C., Valadier, M.: Convex Analysis and Measurable Multifunctions. Springer-Verlag, BerlinHeidelberg-New York (1977)

8. Fechner, W., Sikorska, J.: On the stability of orthogonal additivity. Bull. Polish Acad. Sci. Math. 58, 2330 (2010)

9. Godini, G.: Set-valued Cauchy functional equation. Rev. Roumaine Math. Pures Appl. 20, 1113-1121 (1975)

10. Gudder, S., Strawther, D.: Orthogonality and nonlinear functionals. Bull. Amer. Math. Soc. 80, 946-950 (1974)

11. Heijnen, B., Goovaerts, M.J.: Additivity and premium calculation principles. Blätter der Deutschen Gesellschaft für Versich. Math. 17, 217-223 (1986)

12. Henney, D.: The structure of set-valued additive functions. Portugal. Math. 26, 463-471 (1967)

13. Henney, D.: Properties of set-valued additive functions. Amer. Math. Mon. 75, 384-386 (1968)

14. Hyers, D.H., Isac, G., Rassias, Th.M.: Stability of Functional Equations in Several Variables. Birkhäuser, Boston (1998)

15. Hukuhara, M.: Intégration des applications measurables dont la valeur est un compact convexe. Funkcial. Ekvac. 10, 205-223 (1967)

16. Nikodem, K.: Additive set-valued functions in Hilbert spaces. Rev. Roumaine Math. Pures Appl. 28, 239-242 (1983)

17. Nikodem, K.: Additive selections of additive set-valued functions. Univ. u Novom Sadu Zb. Rad. Prirod.Mat. Fak. Ser. Mat. 18, 143-148 (1988)

18. Nikodem, K.: Set-valued solutions of the Pexider functional equation. Funkcialaj Ekvacioj 31, $227-231$ (1988)

19. Piszczek, M.: Second Hukuhara's derivative and cosine family of linear set valued functions. Ann. Acad. Paed. Cracov. Studia Math. 5, 87-98 (2005)

20. Rådström, H.: An embedding theorem for spaces of convex set. Proc. Amer. Math. Soc. 3, 165-169 (1952)

21. Rådström, H.: One-parameter semigroups of subsets of a real linear space. Ark. Mat. 4, 87-97 (1960)

22. Rätz, J.: On orthogonally additive mappings. Aequationes Math. 28, 35-49 (1985)

23. Rätz, J.: On orthogonally additive mappings, II. Publ. Math. Debrecen 35, 241-249 (1988)

24. Rätz, J.: On orthogonally additive mappings, III. Abh. Math. Sem. Univ. Hamburg 59, $23-33$ (1989)

25. Rätz, J.: Cauchy functional equation problems concerning orthogonality. Aequationes Math. 62, 1-10 (2001)

26. Rätz, J.: Szabó, Gy.: On orthogonally additive mappings, IV. Aequationes Math. 38, 73-85 (1989)

27. Rolewicz, S.: Metric Linear Spaces. Mathematics and its Applications, vol. 20. D. Reidel Publishing Co., Dordrecht-Boston-Lancaster and PWN-Polish Scientific Publishers, Warszawa (1985)

28. Sikorska, J.: On a direct method for proving the Hyers-Ulam stability of functional equations. J. Math. Anal. Appl. 372, 99-109 (2010)

29. Sikorska, J.: Orthogonalities and functional equations. Aequationes Math. (2014). doi:10.1007/s00010014-0288-0

30. Smajdor, W.: Subadditive and subquadratic set-valued functions. Prace Nauk. Uniw. Śląsk. 889. Katowice (1987)

31. Smajdor, W.: On set-valued solutions of a functional equation of Drygas. Aequationes Math. 77, 89-97 (2009)

32. Truesdell, C., Muncaster, R.G.: Fundamentals of Maxwell's Kinetic Theory of a Simple Monatomic Gas. Academic Press, New York (1980)

33. Urbański, R.: A generalization of the Minkowski-Rådström-Hörmander theorem. Bull. Polish Acad. Sci. 24, 709-715 (1976) 